\title{
High- $T_{c}$ superconducting hydrides formed by $\mathrm{LaH}_{24}$ and $\mathrm{YH}_{24}$ cage structures as basic blocks.
}

\author{
Peng Song ${ }^{1}$, Zhufeng $\mathrm{Hou}^{2}$, Pedro Baptista de Castro ${ }^{3,4}$, Kousuke \\ Nakano $^{1,5}$, Kenta Hongo ${ }^{6}$, Yoshihiko Takano ${ }^{3,4}$, Ryo Maezono ${ }^{1}$ \\ ${ }^{1}$ School of Information Science, JAIST, \\ Asahidai 1-1, Nomi, Ishikawa 923-1292, Japan \\ ${ }^{2}$ State Key Laboratory of Structural Chemistry, \\ Fujian Institute of Research on the Structure of Matter, \\ Chinese Academy of Sciences, Fuzhou 350002, China \\ ${ }^{3}$ National Institute for Materials Science, \\ 1-2-1 Sengen, Tsukuba, Ibaraki 305-0047, Japan \\ ${ }^{4}$ University of Tsukuba, 1-1-1 Tennodai, \\ Tsukuba, Ibaraki 305-8577, Japan \\ ${ }^{5}$ International School for Advanced Studies (SISSA), \\ Via Bonomea 265, 34136, Trieste, Italy \\ ${ }^{6}$ Research Center for Advanced Computing Infrastructure, \\ JAIST, Asahidai 1-1, Nomi, Ishikawa 923-1292, Japan
}

(Dated: May 25, 2021)

\begin{abstract}
Based on recent studies regarding high-temperature (high- $T_{c}$ ) La-Y ternary hydrides (e.g., $P \overline{1}-\mathrm{La}_{2} \mathrm{YH}_{12}$, $P m \overline{3} m-\mathrm{LaYH}_{12}$, and $P m \overline{3} m$ - $(\mathrm{La}, \mathrm{Y}) \mathrm{H}_{10}$ with a maximum $\left.T_{c} \sim 253 \mathrm{~K}\right)$, we examined the phase and structural stabilities of the $\left(\mathrm{LaH}_{6}\right)\left(\mathrm{YH}_{6}\right)_{y}$ series as high- $T_{c}$ ternary hydride compositions using a genetic algorithm and $a b$ initio calculations. Our evaluation showed that the $P m \overline{3} m-\mathrm{LaYH}_{12}$ reported in the previous study was unstable during decomposition into $R \overline{3} c-\mathrm{LaH}_{6}+\operatorname{Im} \overline{3} m-\mathrm{YH}_{6}$. We also discovered new crystal structures, namely $C m m m$ $\mathrm{LaYH}_{12}(y=1), R \overline{3} c-\mathrm{LaYH}_{12}(y=1), C m m m-\mathrm{LaY}_{3} \mathrm{H}_{24}(y=3)$, and $R \overline{3}-\mathrm{LaY}_{3} \mathrm{H}_{24}(y=3)$, showing stability against such decomposition. While $R \overline{3} c(y=1)$ and $R \overline{3}(y=3)$ did not exhibit superconductivity owing to the extremely low density of states at the Fermi level, $\mathrm{Cmmm}$ phases exhibited a $T_{c}$ of approximately $140 \mathrm{~K}$ at around $200 \mathrm{GPa}$ owing to the extremely high electron-phonon coupling constant $\left(\lambda=1.876\right.$ for $\left.\mathrm{LaYH}_{12}\right)$. By the twice longer stacking for $\mathrm{Cmmm}-\mathrm{LaY}_{3} \mathrm{H}_{24}$, the coupling constant increased owing to the chemical pressure of Y, leading to a slightly increased $T_{c}$.
\end{abstract}

\section{INTRODUCTION}

Metal hydrides have always been considered ideal candidates for high-temperature (high- $T_{c}$ ) superconductors owing to their ultrahigh phonon vibration frequency. [1, 2] However, realizing superconductivity by keeping them under the metallicity gradient often requires extremely high pressures. [1-6] Therefore, theoretical simulations play an extremely important role in search for novel hydride superconductors. [7- -9$]$ Through theoretical simulations, almost all the predictions for binary hydrides have been completed, and studies have successfully predicted the room-temperature superconductivity of $\mathrm{YH}_{10}$. [10, 11] The successful prediction of roomtemperature superconductivity in ternary $\mathrm{Li}_{2} \mathrm{MgH}_{16}[12]$ and the experimental discovery of room-temperature superconductivity in the $\mathrm{C}-\mathrm{S}-\mathrm{H}$ system [13] have pushed research on superconducting ternary hydrides system to a climax.

However, owing to the diversity of ternary hydrides, the search for new high- $T_{c}$ superconductors appears very slow, and only $10 \sim 20$ potential superconducting hydrides have been identified. [12-32] From the information obtained regarding binary superconductors, the crystal structure signif- icantly influences the $T_{c}$. For example, some ultrahigh$T_{c}$ superconductors often appear in cubic systems such as $\left(F m \overline{3} m-\mathrm{YH}_{10}, F m \overline{3} m-\mathrm{LaH}_{10}\right.$, and $I m \overline{3} m-\mathrm{H}_{3} \mathrm{~S}$, among others. $[10,33,34]$ However, in ternary hydrides, the stability of the cubic structure is very poor, and many ternary systems may exhibit phase decomposition relative to binary hydrides. For example, $\mathrm{CSH}_{7},[28]$ is stable relative to $\mathrm{C}, \mathrm{S}$, and $\mathrm{H}$, but not to binary hydrides $\mathrm{CH}_{4}[35]$ and $\mathrm{H}_{3} \mathrm{~S}$ [34], possibly leading to phase decomposition. Owing to phase decomposition, it is highly unlikely that these compounds can be synthesized experimentally. Therefore, it is extremely important to ensure their stability relative to the constituent elements and binary materials for predicting ternary hydrides.

For searching ternary hydrides, there are two major approaches aiming at obtaining a high $T_{c}$, one focusing on $\mathrm{SH}_{x}$ doped with $\mathrm{La}, \mathrm{Se}, \mathrm{Te}$, and $\mathrm{Cl}$ etc., [19, 36-38] and the other on La- or Y-hydrides doped with metallic elements such as $\mathrm{CaYH}_{12}, \mathrm{CaMgH}_{12}, \mathrm{KScH}_{12}$, and $\mathrm{LaKH}_{12}$. [19, 39, 41] With no significant improvement in $T_{c}$ observed so far in the latter approach, the required pressure to realize superconductivity in these materials is considerably reduced, thereby providing the possibility of their experimental synthesis. Experiments in- 

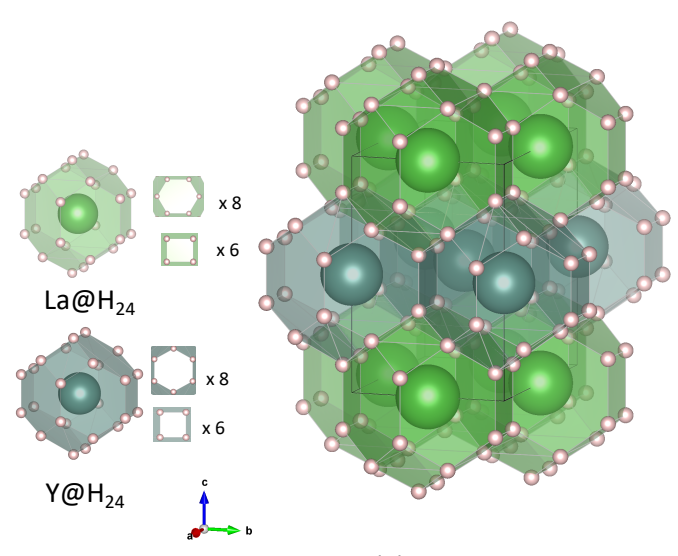

(a) $\mathrm{Cmmm}$ - $\mathrm{LaYH}_{12}$

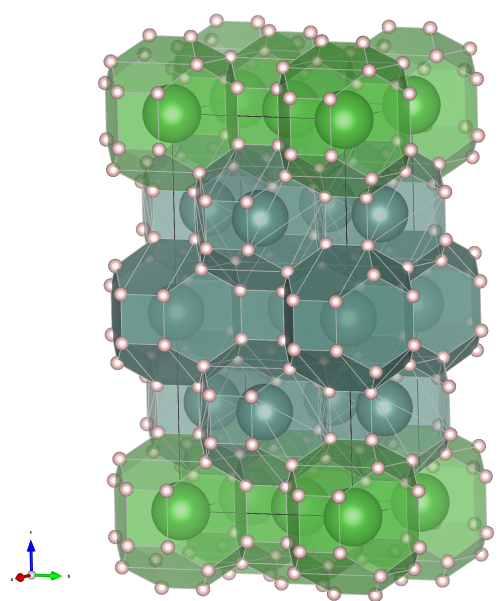

(b) $\mathrm{Cmmm}-\mathrm{LaY}_{3} \mathrm{H}_{24}$

FIG. 1. Clathrate structure of $\mathrm{LaYH}_{12}$ (left panel, at $200 \mathrm{GPa}$ ) and $\mathrm{LaY}_{3} \mathrm{H}_{24}$ (right panel, at $180 \mathrm{GPa}$ ) with Cmmm, consisting of La-centered $\mathrm{H}_{24}$ cages and $\mathrm{Y}$-centered $\mathrm{H}_{24}$ cages. Each $\mathrm{H}_{24}$ cage is surrounded by six squares and eight hexagons.

volving the La-Y-H system report high- $T_{c}$ superconductivity in $(\mathrm{La}, \mathrm{Y}) \mathrm{H}_{10}$. [42]

Based on these experiments, theoretical predictions of superconductivity in $\mathrm{LaYH}_{12}, \mathrm{La}_{2} \mathrm{YH}_{18}, \mathrm{La}_{4} \mathrm{YH}_{30}$ etc. have been also reported. [42] These compositions are referred to as composites of $\left(\mathrm{LaH}_{6}\right)_{x}+\mathrm{YH}_{6}$. The symmetry of their predicted structures is considerably low mainly being $P \overline{1}$ with highest mostly $P m \overline{3} m$ for $\mathrm{LaYH}_{12}$. Examining its decomposition enthalpy revealed that the structure may exhibit phase decomposition under high pressure toward $R \overline{3} c-\mathrm{LaH}_{6}+\operatorname{Im} \overline{3} m$ $\mathrm{YH}_{6}$, being unlikely to be synthesized experimentally. [10, 11] We then determined whether $\mathrm{LaYH}_{12}$ has other structures stable against decomposition based on the USPEX fixed composition method. [7] Composition $\mathrm{LaYH}_{12}$ was also found in our machine learning search based on a gradient boosting tree [41], and it exhibited a high $T_{c}$. When searching more general forms, namely $\left(\mathrm{LaH}_{6}\right)_{x}\left(\mathrm{YH}_{6}\right)_{y}$, we restricted the range by fixing $x=1$ because the preceding work has reported instability along the $x$-axis. Along the $y$-axis, we found $\mathrm{Cmmm}$ $\mathrm{LaYH}_{12}(y=1)$ and $C m m m-\mathrm{LaY}_{3} \mathrm{H}_{24}(y=3)$ exhibiting a high $T_{c} \sim$ of $140 \mathrm{~K}$. These compounds are formed through stacking of $\mathrm{LaH}_{24}$ and $\mathrm{YH}_{24}$ cages, as shown in Fig. 1] A slightly higher $T_{c}$ for $C m m m$ - $\mathrm{LaY}_{3} \mathrm{H}_{24}$ compared to that for $\mathrm{LaYH}_{12}$ can be realized by enhancing the chemical pressure for $\mathrm{Y}$ through further stacking.

\section{RESULTS}

Fig. 2 shows the convex hull of our search for $\mathrm{LaH}_{6}\left(\mathrm{YH}_{6}\right)_{x}$ $(x=1-4)$ structures at 100, 200, and $300 \mathrm{GPa} . \mathrm{LaYH}_{12}(x=1)$ and $\mathrm{LaY}_{3} \mathrm{H}_{24}(x=3)$ are stable phases, whereas other $\mathrm{LaY}_{2} \mathrm{H}_{18}$ and $\mathrm{LaY}_{4} \mathrm{H}_{30}$ structures are likely to be decomposed at the abovementioned pressures . For the stable compositions, namely $\mathrm{LaYH}_{12}$ and $\mathrm{LaY}_{3} \mathrm{H}_{24}$, we compared their relative en- thalpies with those of the candidate structures over the pressure range, as shown in Fig. 3 The plot for $\mathrm{LaYH}_{12}$ indicates that the $P m \overline{3} m$ structure proposed recently [42] exhibits instability toward decomposition into binary compounds, namely $\mathrm{YH}_{6}(\operatorname{Im} \overline{3} m)$ and $\mathrm{LaH}_{6}(R \overline{3} c)$.

For the entire pressure range, more stable structures are predicted as $R \overline{3} c \rightarrow C m m m$ with the transition occurring at approximately $P=140 \mathrm{GPa}$, without instability toward decomposition. For $\mathrm{LaY}_{3} \mathrm{H}_{24}$, stable structures form as $R \overline{3} \rightarrow$ Cmmm, preventing its decomposition either to $\mathrm{LaYH}_{12}+$ $2\left(\mathrm{YH}_{6}\right)$ or $3\left(\mathrm{YH}_{6}\right)+\mathrm{LaH}_{6}$. As explained later, $R \overline{3} c-\mathrm{LaYH}_{12}$ and $R \overline{3}-\mathrm{LaY}_{3} \mathrm{H}_{24}$ exhibit an extremely low density of states (DOS) at the Fermi level, $D\left(\varepsilon_{F}\right)$, leading to a considerably low $T_{c}$. In contrast, $C m m m$ compounds are exhibit a higher $T_{c}$, and their structures are shown in Fig. 1. Detailed geometries for each structure are provided in Table $\Pi$ in the Supplemental Information (S.I.).

As observed in the Fig. 1, the structures are mostly clathrate structures formed by units with $\mathrm{La}_{0} \mathrm{H}_{24}$ and $\mathrm{Y} @ \mathrm{H}_{24}$ cage structures. Several other clathrate superconductors have been identified, [10-12, [21, 43-45] and hence it would be plausible for this structure to exhibit superconductivity. Different periodicities of the La- and Y-layers along the $c$-direction correspond to $\mathrm{LaYH}_{12}[(\mathrm{La} / \mathrm{Y})(\mathrm{La} / \mathrm{Y}) \cdots]$ and $\mathrm{LaY}_{3} \mathrm{H}_{24}[(\mathrm{La} / \mathrm{Y} / \mathrm{Y} / \mathrm{Y})(\mathrm{La} / \mathrm{Y} / \mathrm{Y} / \mathrm{Y}) \cdots]$, respectively.

Fig. 4 shows the phonon dispersions evaluated for the clathrate Cmmm $\mathrm{LaYH}_{12}$. We confirmed no imaginary modes, thus confirming its structural stability. The phonon dispersions for other structures are given in the S.I., confirming that all the candidates predicted from Fig. 3 namely $\mathrm{Cmmm-}$ $\mathrm{LaYH}_{12}, C m m m-\mathrm{LaY}_{3} \mathrm{H}_{24} R \overline{3} c-\mathrm{LaYH}_{12}$, and $R \overline{3}-\mathrm{LaY}_{3} \mathrm{H}_{24}$, have no imaginary modes. In contrast, $P m \overline{3} m-\mathrm{LaYH}_{12}$ proposed recently [42] exhibits imaginary modes, as shown in Fig. 5, consistent with the observations from Fig. 3 in terms 


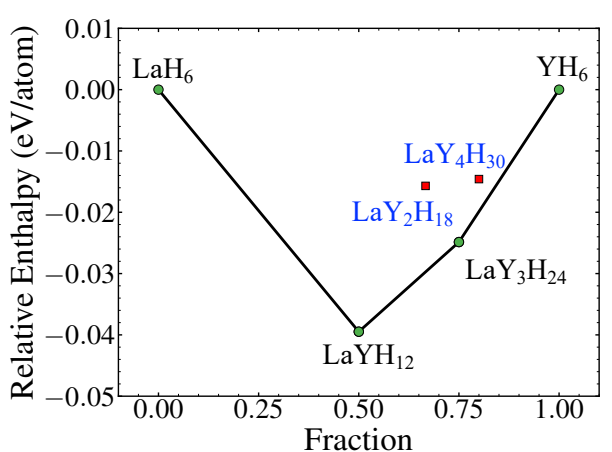

(a) $100 \mathrm{GPa}$

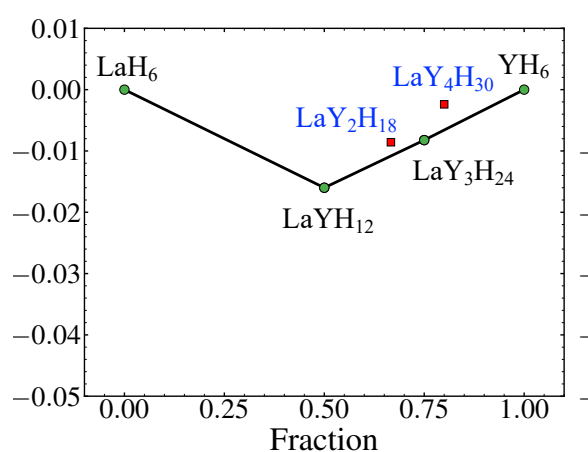

(b) $200 \mathrm{GPa}$

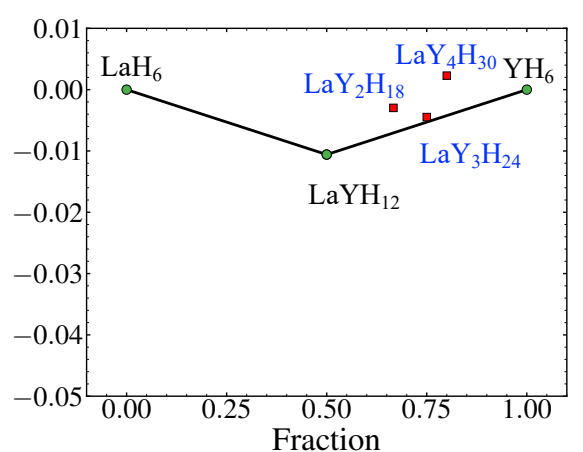

(c) $300 \mathrm{GPa}$

FIG. 2. Convex hull of La-Y-H at pressures of $100 \mathrm{GPa}, 200 \mathrm{GPa}$, and $300 \mathrm{GPa}$.

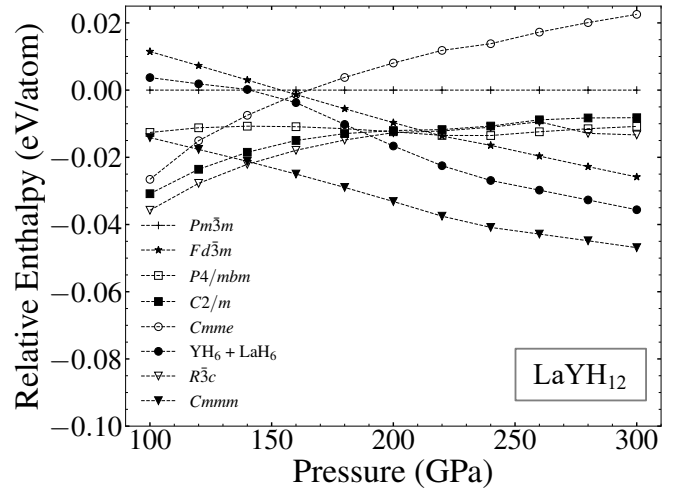

(a) $\mathrm{LaYH}_{12}$

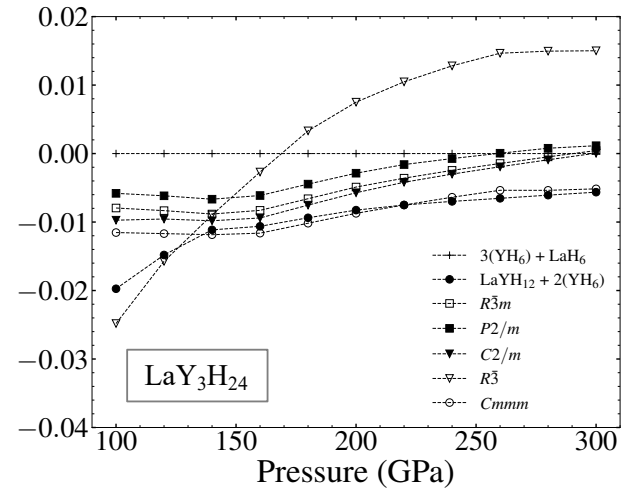

(b) $\mathrm{LaY}_{3} \mathrm{H}_{24}$

FIG. 3. Comparisons of candidate structure enthalpies for $\mathrm{LaYH}_{12}$ [panel (a)] and $\mathrm{LaY}_{3} \mathrm{H}_{24}$ [panel (b)]. Such structures giving higher values than those of ' $\mathrm{YH}_{6}+\mathrm{LaH}_{6}$ ' (for the left panel), '3( $\left.\mathrm{YH}_{6}\right)+\mathrm{LaH}_{6}$ ' (for the right), and ' $\mathrm{LaYH}_{12}+2\left(\mathrm{YH}_{6}\right.$ )' (for the right), are predicted to exhibit instability toward decomposition into these binary compounds.

of the instability.

Applying the Allen-Dynes formalism, [46-48] we estimated $T_{c}$ as summarized in Table I] For $\mathrm{LaYH}_{12}, T_{c}$ is in the $130.6 \sim 140.55[\mathrm{~K}]$ range, depending on the choice of parameter $\mu=0.10 \sim 0.13$ for the Coulomb interaction. A slightly higher $T_{c}$ realized for $\mathrm{LaY}_{3} \mathrm{H}_{24}$ is attributed to the higher $D\left(\varepsilon_{F}\right)$ due to the compressed chemical pressure for the $\mathrm{Y}$-site with the $(\mathrm{La} / \mathrm{Y} / \mathrm{Y} / \mathrm{Y})$ stacking structure, compared to the $(\mathrm{La} / \mathrm{Y})(\mathrm{La} / \mathrm{Y})$ periodicity.

\section{DISCUSSIONS}

As observed for $F d \overline{3} m-\mathrm{Li}_{2} \mathrm{MgH}_{16}$, a room-temperature superconductor [12], the key factors for achieving a high $T_{c}$ are its high DOS and high phonon frequency, which can be measured by $\lambda$ and $\omega_{\log }$, respectively. $\lambda=2$ would be the typical criterion for realizing superconductivity $(\lambda=3.35$ for $F d \overline{3} m$ -

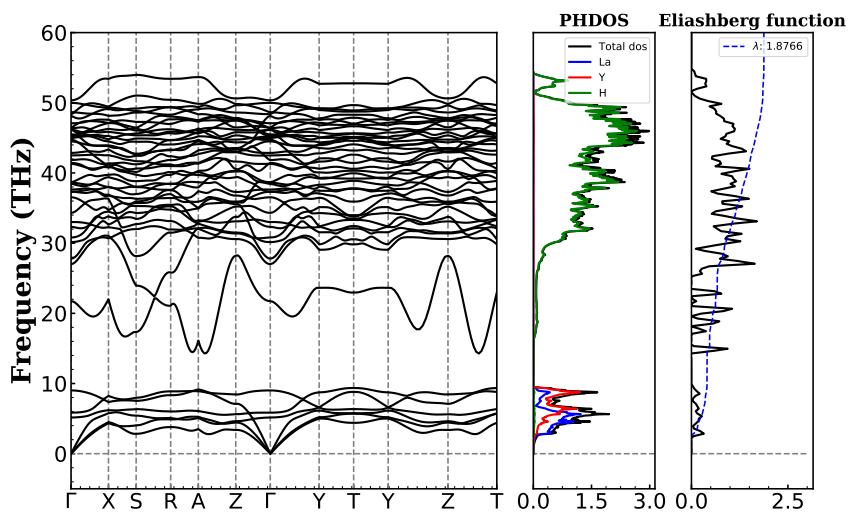

FIG. 4. Phonon dispersions, projected phonon densities of states, and Eliashberg spectral function for $\mathrm{Cmmm}-\mathrm{LaYH}_{12}$ clathrate at $200 \mathrm{GPa}$. 
TABLE I. $T_{c}$ (expressed in [K]) estimated using the Allen-Dynes formula through ab initio phonon calculations for $\mathrm{LaYH}_{12}$ and $\mathrm{LaY}_{3} \mathrm{H}_{24}$ at each pressure. $\lambda$ and $\omega_{\log }$ (expressed in $\left.[\mathrm{K}]\right)$ are the parameters appearing in the formula. The values of $D\left(\varepsilon_{F}\right)$ are are expressed in [states/eV/atom].

\begin{tabular}{rcrrrl}
\hline & $P[\mathrm{GPa}]$ & $\lambda$ & $\omega_{\log }$ & $D\left(\varepsilon_{F}\right)$ & $T_{c}(\mu=0.1-0.13)$ \\
\hline $\mathrm{LaYH}_{12}$ & 200 & 1.876 & 1022.54 & 0.097 & $140.55-130.61$ \\
$\mathrm{LaYH}_{12}$ & 250 & 1.618 & 1051.56 & 0.093 & $128.42-117.96$ \\
$\mathrm{LaY}_{3} \mathrm{H}_{24}$ & 180 & 2.452 & 891.49 & 0.100 & $145.31-137.11$ \\
\hline
\end{tabular}

$\left.\mathrm{Li}_{2} \mathrm{MgH}_{16}\right)$. [12]

In the S.I., we have provided the band structures and Fermi surfaces of $R \overline{3} c$ - $\mathrm{LaYH}_{12}$ and $R \overline{3} c$ - $\mathrm{LaYH}_{12}$ (Figs. 10 and 11 . The Fermi surfaces and the DOS values on these surfaces are extremely small and are insufficient to achieve superconductivity.

In contrast, for the $C m m m$ structure, sufficiently large Fermi surfaces and high DOS values lead to higher $\lambda$, thus resulting in superconductivity. Examining the phonon dispersions and Eliashberg spectral functions (Figs. 4, 8 and 97 reveals that higher $\lambda$ values can be attributed to the contribution from the phonon branches appearing at higher ranges $(>10 \mathrm{THz})$, amounting to $96.2 \%$. These branches are formed by the vibration of hydrogen atoms, whereas those at lower ranges $(<$ $10 \mathrm{THz}$ ) by La and Y, negligibly contributing to $\lambda$.

Applying higher pressures $(200 \rightarrow 250 \mathrm{GPa})$ to $\mathrm{Cmmm}$ $\mathrm{LaY}_{3} \mathrm{H}_{24}$ increases its phonon frequencies for the branches at higher ranges (>10 THz), as observed in Figs. 4 and 8 . This trend is reflected as the increased $\omega_{\log }$ (Table II), contributing toward increasing the $T_{c}$. However, this trend disappears when the DOS (around 4.2\%) decreases owing to the applied pressure, leading to a reduced $\lambda$ value. This decrease is more dominant than the increase in $\omega_{\text {log }}$, and hence the applied pressure eventually lowers the $T_{c}$, as observed in Table 1

The slightly higher $T_{c}$ determined for $\mathrm{LaY}_{3} \mathrm{H}_{24}$ is attributed to the enhanced $\lambda$, as observed in Table I] In this case too, changes in $\omega_{\log }$ and $\lambda$ exhibit a cancelling relationship, causing only a slight increase in the $T_{c}$. Applying further pressure would mean increasing $\lambda$ further for achieving a higher $T_{c}$; however, according to the convex hull in our analysis, Cmmm- $\mathrm{LaY}_{3} \mathrm{H}_{24}$ exhibits instability toward phase decomposition above $220 \mathrm{GPa}$.

$P m \overline{3} m-\mathrm{LaYH}_{12}$ exhibits a higher $T_{c}(203 \mathrm{~K}$ at $180 \mathrm{GPa})$ [42] However, for this structure, the imaginary phonon modes appeared as shown in Fig. 5 implying structural instability at least within the extent of the harmonic approximation. As shown in Fig. 3, another structure, $F d \overline{3} m-\mathrm{LaYH}_{12}$, is predicted to be more stable than $P m \overline{3} m$. However, for both structures, the imaginary modes are found, without vanishing even on applying further pressure.

$P m \overline{3} m-\mathrm{LaYH}_{12}$ can be regarded as a compound based on the $\operatorname{Im} \overline{3} m-\mathrm{YH}_{6}$ dimer with La substituting for one Y (Fig. 6 . The $\operatorname{Im} \overline{3} m-\mathrm{YH}_{6}$ actually appears as a stable structure in the convex-hull analysis given in Fig. 2. Our prediction, Cmmm$\mathrm{LaYH}_{12}$, is actually a distorted structure of $P m \overline{3} m-\mathrm{LaYH}_{12}$,

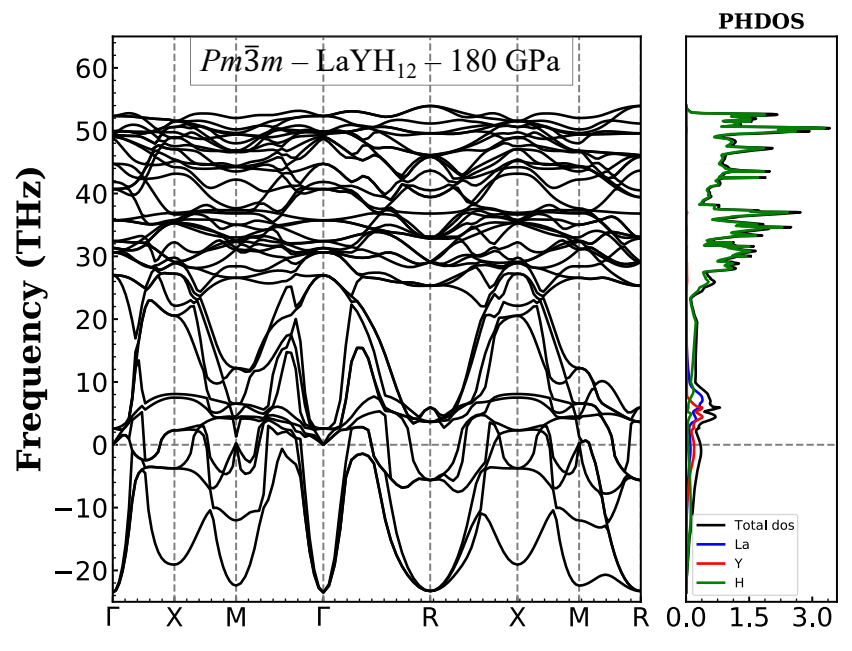

FIG. 5. Phonon dispersions and projected phonon densities of states for $P m \overline{3} m-\mathrm{LaYH}_{12}$ at $180 \mathrm{GPa}$.

showing considerably similar X-ray diffraction (XRD) peak patterns as well as that of $F d \overline{3} m$, as shown in Fig. 2. Furthermore, the possibility of the structural transition between $C m m m$ and $P m \overline{3} m$ at higher temperatures is pointed out. [12]

\section{CONCLUSION}

In summary, we investigated the possible crystal structures for $\left(\mathrm{LaH}_{6}\right)\left(\mathrm{YH}_{6}\right)_{y}$ compounds as candidates for forming ternary hydrides to achieve a high $T_{c}$ using a generic algorithm implemented in USPEX. We determined the thermal stabilities at $y=1\left(\mathrm{LaYH}_{12}\right)$ and $y=3\left(\mathrm{LaY}_{3} \mathrm{H}_{24}\right)$. A recently reported structure, $\operatorname{Pm} \overline{3} m$, appeared unstable toward the decomposition, i.e., $\mathrm{LaYH}_{12}(P m \overline{3} m) \rightarrow \mathrm{LaH}_{6}(R \overline{3} c)$ $+\mathrm{YH}_{6}(\operatorname{Im} \overline{3} m)$. However, we discovered other new stable structures that did not exhibit decomposition, namely $\mathrm{Cmmm-}$ $\mathrm{LaYH}_{12}, R \overline{3} c-\mathrm{LaYH}_{12}, C m m m-\mathrm{LaY}_{3} \mathrm{H}_{24}$, and $R \overline{3}-\mathrm{LaY}_{3} \mathrm{H}_{24}$. Among these, the compounds with $\mathrm{Cmmm}$ clathrate structures exhibited a $T_{c} \sim 130[\mathrm{~K}]$ at approximately $P=200 \mathrm{GPa}$, as estimated using the Allen-Dynes formalism. 


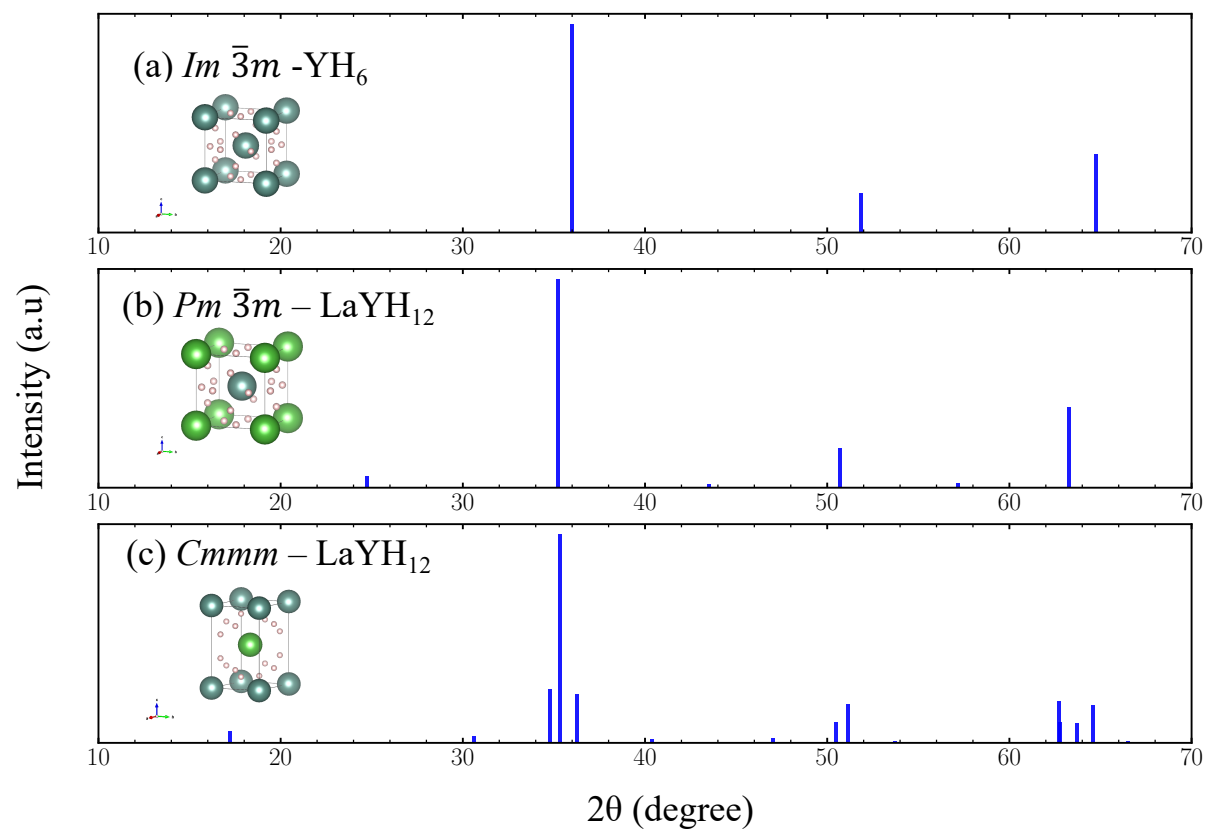

FIG. 6. Structures and the XRD patterns of (a) $I m \overline{3} m-\mathrm{YH}_{6}$, (b) $P m \overline{3} m-\mathrm{LaYH}_{12}$, and (c) Cmmm-LaYH12 12 .

\section{METHODS}

For the structural predictions, we used the USPEX code [7] combined with the $a b$ initio kernel in the Vienna ab initio simulation package (VASP). [49-52] For example, the $\mathrm{LaYH}_{12}$ composition randomly generated 400 structures ranging from monomers to tetramers with a $\mathrm{LaYH}_{12}$ unit as the "initial generation." Each generation evolved 100 structures with $40 \%$ heredity, $40 \%$ randomness, $10 \%$ softmutation, and $10 \%$ transmutation. When no further evolution occurred for more than 10 generations, the final structure was identified as the candidate crystal structure of the composition. For the identified candidates, we performed $a b$ initio geometrical optimizations using the Perdew-Burke-Ernzerhof (GGA-PBE) functional for the exchange-correlation functional. [53]

For the most stable candidates $[R \overline{3} c(100-140 \mathrm{GPa})$ and Cmmm (140-300 GPa) of $\mathrm{LaYH}_{12}$, for example], we performed $a b$ intio phonon calculations to evaluate their structural stabilities and $T_{c}$ using the Allen-Dynes formalism, [4648] implemented in Quantum Espresso. [46] Computational conditions such as the cutoff energy of the plane wave ba- sis set, sizes of the grid-mesh over the Brillouin zone etc. were determined such that the energies sufficiently converged in their dependences. We finally used an $8 \times 8 \times 5 k$-mesh used in the self-consistent field convergence assisted by the Marzari-Vanderbilt smearing scheme. [54] The final energy values were estimated through extrapolation of the smearing parameter toward zero. Mesh sizes for the phonon calculations were $16 \times 16 \times 10$ for the $k$ - and $4 \times 4 \times 2$ for the $q$-mesh.

\section{ACKNOWLEDGMENTS}

The computations in this work have been performed using the facilities of Research Center for Advanced Computing Infrastructure (RCACI) at JAIST. K.H. is grateful for financial support from the HPCI System Research Project (Project ID: hp190169) and MEXT-KAKENHI (JP16H06439, JP17K17762, JP19K05029, and JP19H05169). R.M. is grateful for financial supports from MEXTKAKENHI (21K03400 and 19H04692), and the Air Force Office of Scientific Research (AFOSR-AOARD/FA2386-171-4049;FA2386-19-1-4015)
[1] N. Ashcroft, Phys. Rev. Lett. 92, 187002 (2004).

[2] N. W. Ashcroft, Phys. Rev. Lett. 21, 1748 (1968).

[3] M. Eremets, I. Trojan, S. Medvedev, J. Tse, and Y. Yao, Science 319, 1506 (2008).

[4] A. Drozdov, M. Eremets, I. Troyan, V. Ksenofontov, and S. I. Shylin, Nature 525, 73 (2015).
[5] D. Szczȩśniak and T. Zemła, Supercond. Sci. Technol. 28, 085018 (2015).

[6] E. Snider, N. Dasenbrock-Gammon, R. McBride, X. Wang, N. Meyers, K. V. Lawler, E. Zurek, A. Salamat, and R. P. Dias, Phys. Rev. Lett. 126, 117003 (2021).

[7] C. W. Glass, A. R. Oganov, and N. Hansen, Comput. Phys. Commun. 175, 713 (2006). 
[8] Y. Wang, J. Lv, L. Zhu, and Y. Ma, Comput. Phys. Commun. 183, 2063 (2012).

[9] D. C. Lonie and E. Zurek, Comput. Phys. Commun. 182, 372 (2011).

[10] F. Peng, Y. Sun, C. J. Pickard, R. J. Needs, Q. Wu, and Y. Ma, Phys. Rev. Lett. 119, 107001 (2017).

[11] C. Heil, S. Di Cataldo, G. B. Bachelet, and L. Boeri, Phys. Rev. B 99, 220502 (2019).

[12] Y. Sun, J. Lv, Y. Xie, H. Liu, and Y. Ma, Phys. Rev. Lett. 123, 097001 (2019).

[13] E. Snider, N. Dasenbrock-Gammon, R. McBride, M. Debessai, H. Vindana, K. Vencatasamy, K. V. Lawler, A. Salamat, and R. P. Dias, Nature 586, 373 (2020).

[14] Y. Ma, D. Duan, Z. Shao, H. Yu, H. Liu, F. Tian, X. Huang, D. Li, B. Liu, and T. Cui, Phys. Rev. B 96, 144518 (2017).

[15] Y. Ma, D. Duan, Z. Shao, D. Li, L. Wang, H. Yu, F. Tian, H. Xie, B. Liu, and T. Cui, Phys. Chem. Chem. Phys. 19, 27406 (2017).

[16] D. Li, Y. Liu, F.-B. Tian, S.-L. Wei, Z. Liu, D.-F. Duan, B.-B. Liu, and T. Cui, Front. Phys. 13, 137107 (2018).

[17] X. Liang, S. Zhao, C. Shao, A. Bergara, H. Liu, L. Wang, R. Sun, Y. Zhang, Y. Gao, Z. Zhao, et al., Phys. Rev. B 100, 184502 (2019).

[18] M. Rahm, R. Hoffmann, and N. Ashcroft, J. Am. Chem. Soc. 139, 8740 (2017).

[19] X. Liang, A. Bergara, L. Wang, B. Wen, Z. Zhao, X.-F. Zhou, J. He, G. Gao, and Y. Tian, Phys. Rev. B 99, 100505 (2019).

[20] Y. K. Wei, L. Q. Jia, Y. Y. Fang, L. J. Wang, Z. X. Qian, J. N. Yuan, G. Selvaraj, G. F. Ji, and D. Q. Wei, Int. J. Quantum Chem. , e26459 (2020).

[21] H. Xie, D. Duan, Z. Shao, H. Song, Y. Wang, X. Xiao, D. Li, F. Tian, B. Liu, and T. Cui, J. Phys.: Condens. Matter 31, 245404 (2019).

[22] C. Kokail, W. von der Linden, and L. Boeri, Phys. Rev. Mater. 1, 074803 (2017).

[23] Z. Shao, D. Duan, Y. Ma, H. Yu, H. Song, H. Xie, D. Li, F. Tian, B. Liu, and T. Cui, npj Comput. Mater. 5, 1 (2019).

[24] J. Zhang, J. M. McMahon, A. R. Oganov, X. Li, X. Dong, H. Dong, and S. Wang, Phys. Rev. B 101, 134108 (2020).

[25] P. Zhang, Y. Sun, X. Li, J. Lv, and H. Liu, Phys. Rev. B 102, 184103 (2020).

[26] S. Di Cataldo, W. von der Linden, and L. Boeri, Phys. Rev. B 102, 014516 (2020).

[27] X. Guo, R.-L. Wang, H.-L. Chen, W.-C. Lu, K. Ho, and C. Wang, Phys. Lett. A 384, 126189 (2020).

[28] W. Cui, T. Bi, J. Shi, Y. Li, H. Liu, E. Zurek, and R. J. Hemley, Phys. Rev. B 101, 134504 (2020).

[29] H.-Y. Lv, S.-Y. Zhang, M.-H. Li, Y.-L. Hai, N. Lu, W.-J. Li, and G.-H. Zhong, Phys. Chem. Chem. Phys. 22, 1069 (2020).

[30] Y. Yan, T. Bi, N. Geng, X. Wang, and E. Zurek, J. Phys. Chem. Lett. 11, 9629 (2020).

[31] T. Muramatsu, W. K. Wanene, M. Somayazulu, E. Vinitsky, D. Chandra, T. A. Strobel, V. V. Struzhkin, and R. J. Hemley, J. Phys. Chem. C 119, 18007 (2015).

[32] D. Meng, M. Sakata, K. Shimizu, Y. Iijima, H. Saitoh, T. Sato, S. Takagi, and S.-i. Orimo, Phys. Rev. B 99, 024508 (2019).

[33] M. Somayazulu, M. Ahart, A. K. Mishra, Z. M. Geballe, M. Baldini, Y. Meng, V. V. Struzhkin, and R. J. Hemley, Phys. Rev. Lett. 122, 027001 (2019).

[34] I. Errea, M. Calandra, C. J. Pickard, J. Nelson, R. J. Needs, Y. Li, H. Liu, Y. Zhang, Y. Ma, and F. Mauri, Phys. Rev. Lett. 114, 157004 (2015).

[35] G. Gao, A. R. Oganov, Y. Ma, H. Wang, P. Li, Y. Li, T. Iitaka, and G. Zou, J. Chem. Phys. 133, 144508 (2010).
[36] B. Liu, W. Cui, J. Shi, L. Zhu, J. Chen, S. Lin, R. Su, J. Ma, K. Yang, M. Xu, et al., Phys. Rev. B 98, 174101 (2018).

[37] P.-H. Chang, S. Silayi, D. Papaconstantopoulos, and M. Mehl, J. Phys. Chem. Solids 139, 109315 (2020).

[38] A. Nakanishi, T. Ishikawa, and K. Shimizu, J. Phys. Soc. Jpn. 87, 124711 (2018).

[39] W. Sukmas, P. Tsuppayakorn-aek, U. Pinsook, and T. Bovornratanaraks, J. Alloys Compd. 849, 156434 (2020).

[40] T. Ishikawa, T. Miyake, and K. Shimizu, Phys. Rev. B 100, 174506 (2019).

[41] P. Song, Z. Hou, P. Castro, K. Nakano, K. Hongo, Y. Takano, and R. Maezono, arXiv preprint arXiv:2103.00193 (2021).

[42] D. V. Semenok, I. A. Troyan, A. G. Kvashnin, A. G. Ivanova, M. Hanfland, A. V. Sadakov, O. A. Sobolevskiy, K. S. Pervakov, A. G. Gavriliuk, I. S. Lyubutin, et al., arXiv preprint arXiv:2012.04787 (2020).

[43] H. Wang, S. T. John, K. Tanaka, T. Iitaka, and Y. Ma, PNAS 109, 6463 (2012).

[44] X.-h. Wang, F.-w. Zheng, Z.-w. Gu, F.-1. Tan, J.-h. Zhao, C.-1. Liu, C.-w. Sun, J. Liu, and P. Zhang, ACS Omega (2021).

[45] N. P. Salke, M. M. D. Esfahani, Y. Zhang, I. A. Kruglov, J. Zhou, Y. Wang, E. Greenberg, V. B. Prakapenka, J. Liu, A. R. Oganov, et al., Nat. Commun. 10, 1 (2019).

[46] P. Giannozzi, S. Baroni, N. Bonini, M. Calandra, R. Car, C. Cavazzoni, D. Ceresoli, G. L. Chiarotti, M. Cococcioni, I. Dabo, et al., J. Phys. Condens. Matter. 21, 395502 (2009).

[47] K. Nakano, K. Hongo, and R. Maezono, Inorg. Chem. 56, 13732 (2017).

[48] K. Nakano, K. Hongo, and R. Maezono, Sci. Rep. 6, 1 (2016).

[49] G. Kresse and J. Hafner, Phys. Rev. B 47, 558 (1993).

[50] G. Kresse and J. Hafner, Phys. Rev. B 49, 14251 (1994).

[51] G. Kresse and J. Furthmüller, Comput. Mater. Sci. 6, 15 (1996).

[52] G. Kresse and J. Furthmüller, Phys. Rev. B 54, 11169 (1996).

[53] J. P. Perdew, K. Burke, and M. Ernzerhof, Phys. Rev. Lett. 77, 3865 (1996).

[54] N. Marzari, D. Vanderbilt, A. De Vita, and M. Payne, Phys. Rev. Lett. 82, 3296 (1999). 


\section{SUPPLEMENTAL INFORMATION}

For each compound reported in the main text, we provide the detailed geometries [Table [I] ], Phonon dispersions and Eliashberg spectral functions [Figs. 77,9], and electronic band structures and Fermi surfaces [Figs. 10 13]. To show the bonding characteristics of the hydrogen atoms in each compound, we also provide the results of the electron localization function (ELF) [Figs. 14 and 15], and the crystal orbital Hamilton population (COHP) [Fig. [16]. 
TABLE II. Crystal structures of $\mathrm{LaYH}_{12}$ and $\mathrm{LaY}_{3} \mathrm{H}_{24}$ predicted at each pressure $(P)$. Lattice parameters $(a, b$, and $c)$ are given in units of $\AA$.

\begin{tabular}{|c|c|c|c|c|c|c|c|}
\hline & & \multirow{2}{*}{$P(\mathrm{GPa})$} & \multirow{2}{*}{ Lattice parameters } & \multicolumn{4}{|c|}{ Atomic coordinates (fractional) } \\
\hline & & & & Atoms & $x$ & $y$ & $z$ \\
\hline \multirow{4}{*}{$\mathrm{LaYH}_{12}$} & \multirow{4}{*}{$R \overline{3} c$} & \multirow{4}{*}{100} & $a=b=5.389$ & $\mathrm{La}(6 b)$ & 0.00000 & 0.00000 & 0.00000 \\
\hline & & & $c=13.674$ & $\mathrm{Y}(6 a)$ & 0.00000 & 0.00000 & 0.25000 \\
\hline & & & $\alpha=\beta=90^{\circ}$ & $\mathrm{H}(36 f)$ & 0.00181 & 0.23464 & 0.36866 \\
\hline & & & $\gamma=120^{\circ}$ & $\mathrm{H}(36 f)$ & 0.00492 & 0.27691 & 0.60940 \\
\hline \multirow{5}{*}{$\mathrm{LaYH}_{12}$} & \multirow{5}{*}{$\mathrm{Cmmm}$} & \multirow{5}{*}{200} & \multirow{5}{*}{$\begin{aligned} a & =3.617 \\
b & =4.956 \\
c & =5.156 \\
\alpha=\beta=\gamma & =90^{\circ}\end{aligned}$} & $\mathrm{La}(2 c)$ & 0.00000 & 0.50000 & 0.50000 \\
\hline & & & & $\mathrm{Y}(2 a)$ & 0.00000 & 0.00000 & 0.00000 \\
\hline & & & & $\mathrm{H}(8 n)$ & 0.00000 & 0.11589 & 0.35519 \\
\hline & & & & $\mathrm{H}(8 n)$ & 0.00000 & 0.38205 & 0.11708 \\
\hline & & & & $\mathrm{H}(8 m)$ & 0.25000 & 0.25000 & 0.23317 \\
\hline \multirow{7}{*}{$\mathrm{LaY}_{3} \mathrm{H}_{24}$} & \multirow{7}{*}{$R \overline{3}$} & \multirow{7}{*}{100} & \multirow{7}{*}{$\begin{array}{r}a=b=5.324 \\
c=13.569 \\
\alpha=\beta=90^{\circ} \\
\gamma=120^{\circ}\end{array}$} & $\mathrm{La}(3 a)$ & 0.00000 & 0.00000 & 0.00000 \\
\hline & & & & $\mathrm{Y}(6 c)$ & 0.00000 & 0.00000 & 0.24782 \\
\hline & & & & $\mathrm{Y}(3 b)$ & 0.00000 & 0.00000 & 0.50000 \\
\hline & & & & $\mathrm{H}(18 f)$ & 0.00154 & 0.26668 & 0.60934 \\
\hline & & & & $\mathrm{H}(18 f)$ & 0.00245 & 0.23293 & 0.36741 \\
\hline & & & & $\mathrm{H}(18 f)$ & 0.00465 & 0.75887 & 0.87000 \\
\hline & & & & $\mathrm{H}(18 f)$ & 0.01123 & 0.72593 & 0.11041 \\
\hline \multirow{9}{*}{$\mathrm{LaY}_{3} \mathrm{H}_{24}$} & \multirow{9}{*}{ Cmmm } & \multirow{9}{*}{180} & \multirow{9}{*}{$\begin{array}{r}a=3.621 \\
b=5.013 \\
c=10.239 \\
\alpha=\beta=\gamma=90^{\circ}\end{array}$} & $\mathrm{La}(2 a)$ & 0.00000 & 0.00000 & 0.00000 \\
\hline & & & & $\mathrm{Y}(4 l)$ & 0.00000 & 0.50000 & 0.25606 \\
\hline & & & & $\mathrm{Y}(2 d)$ & 0.00000 & 0.00000 & 0.50000 \\
\hline & & & & $\mathrm{H}(8 n)$ & 0.00000 & 0.11988 & 0.19483 \\
\hline & & & & $\mathrm{H}(8 n)$ & 0.00000 & 0.12176 & 0.31540 \\
\hline & & & & $\mathrm{H}(8 n)$ & 0.00000 & 0.37658 & 0.43798 \\
\hline & & & & $\mathrm{H}(8 n)$ & 0.00000 & 0.38314 & 0.07100 \\
\hline & & & & $\mathrm{H}(8 m)$ & 0.25000 & 0.25000 & 0.13624 \\
\hline & & & & $\mathrm{H}(8 m)$ & 0.25000 & 0.25000 & 0.37798 \\
\hline
\end{tabular}
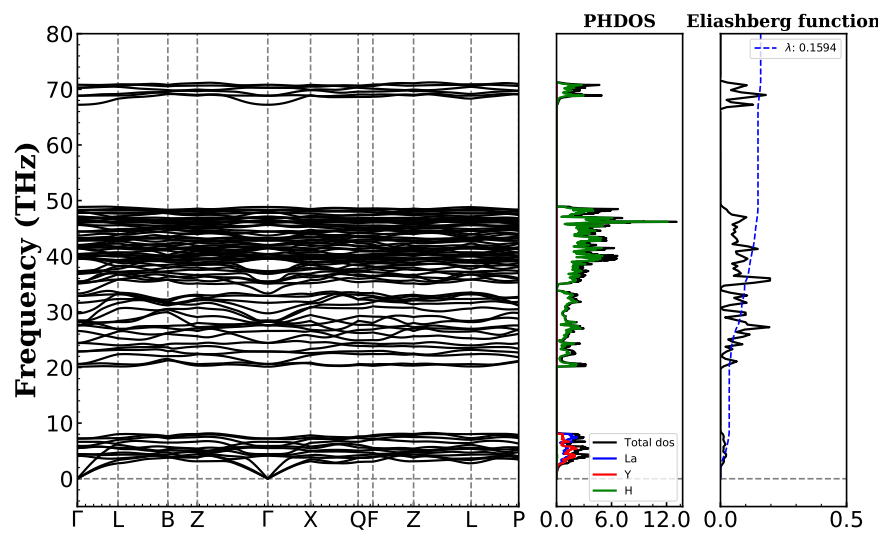

FIG. 7. Phonon dispersions and projected phonon densities of states for $R \overline{3} c-\mathrm{LaYH}_{12}$ clathrate at $100 \mathrm{GPa}$. 

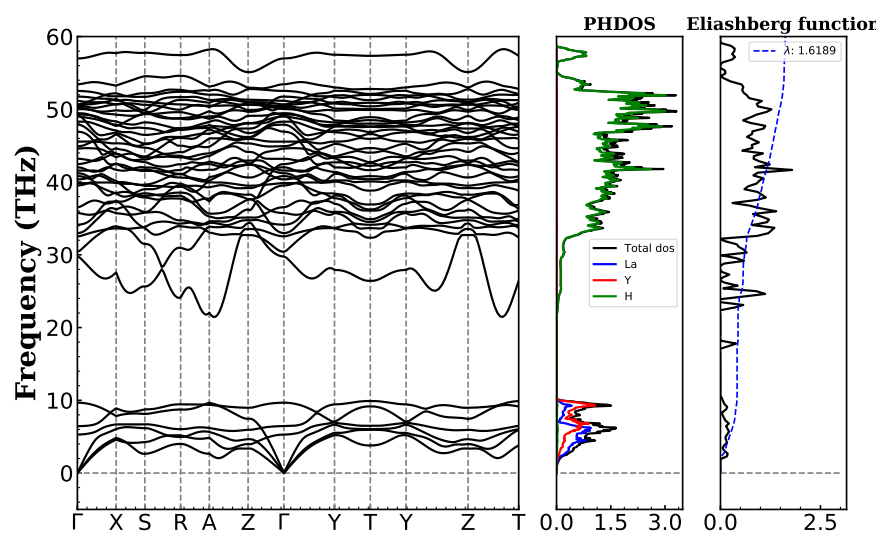

FIG. 8. Phonon dispersions, projected phonon densities of states, and Eliashberg spectral function for $\mathrm{Cmmm}_{\mathrm{LaYH}} \mathrm{La}_{12} \mathrm{clathrate}$ at $250 \mathrm{GPa}$.
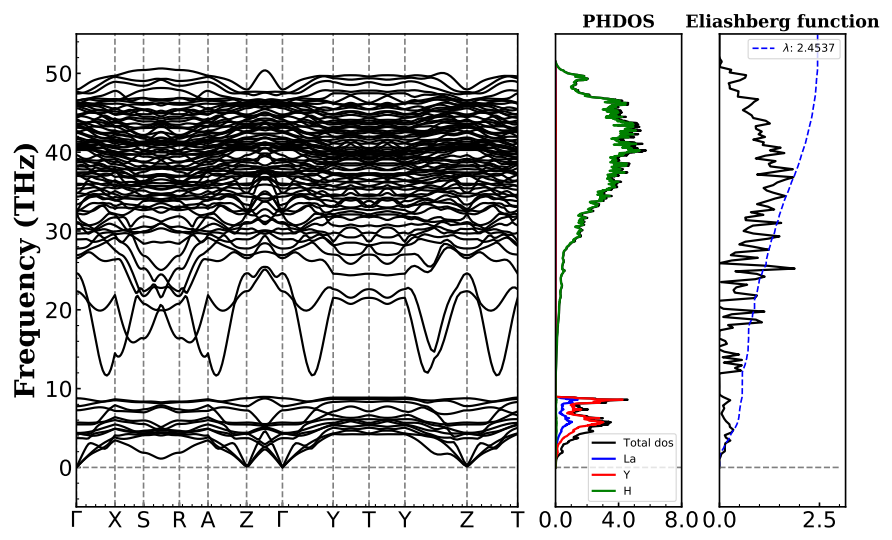

FIG. 9. Phonon dispersions, projected phonon densities of states, and Eliashberg spectral function for $\mathrm{Cmmm}^{-\mathrm{LaY}} \mathrm{H}_{24} \mathrm{clathrate}$ at $180 \mathrm{GPa}$. 

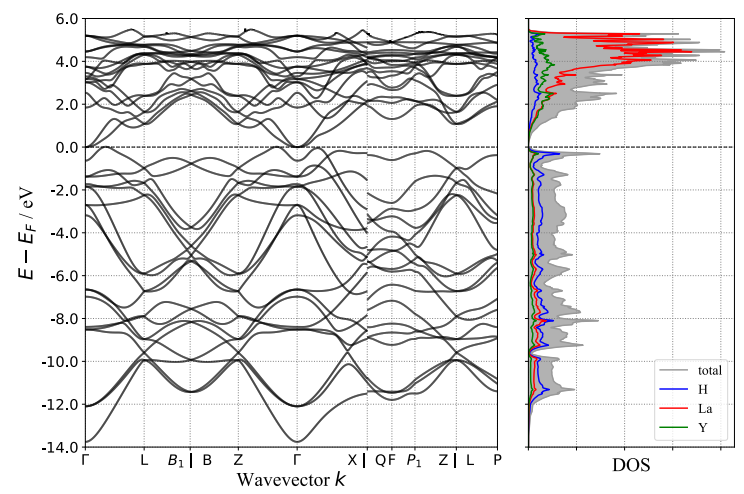

(a)

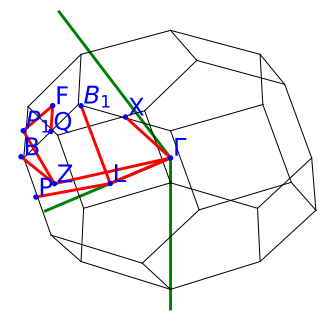

(b)

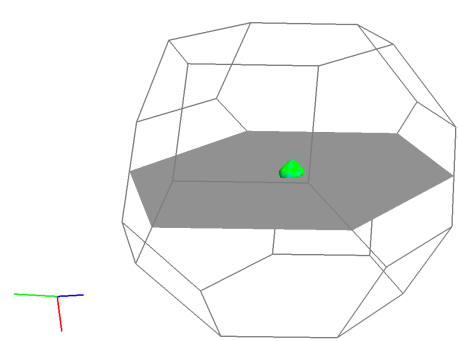

(c)

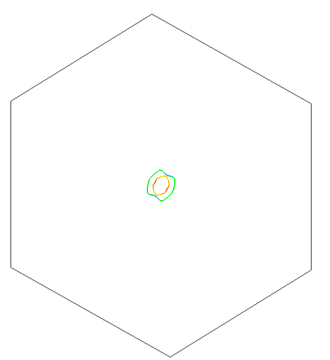

(d)

FIG. 10. Band structure, DOS, and Fermi surface of $R \overline{3} c-\mathrm{LaYH}_{12}$.

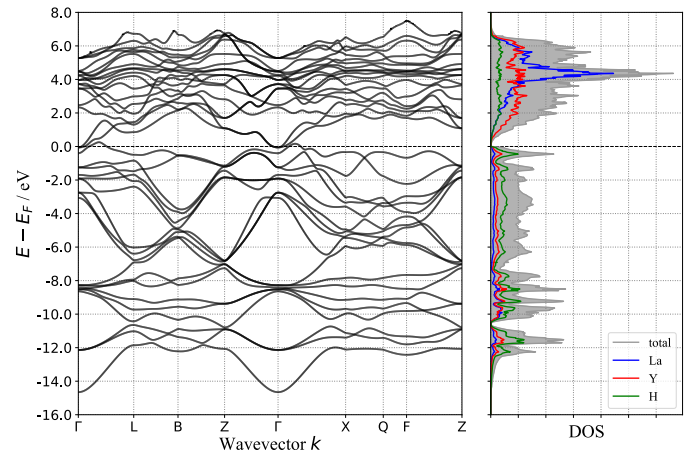

(a)

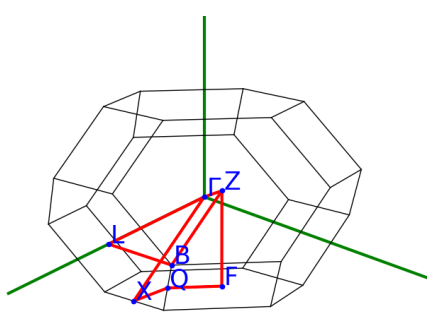

(b)

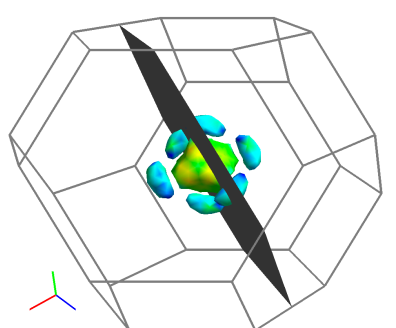

(c)

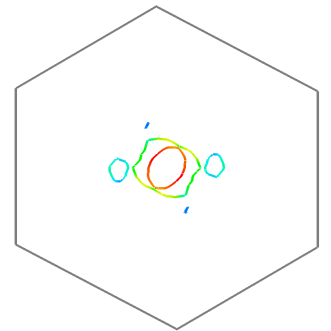

(d)

FIG. 11. Band structure, DOS, and Fermi surface of $R \overline{3}-\mathrm{LaY}_{3} \mathrm{H}_{24}$. 

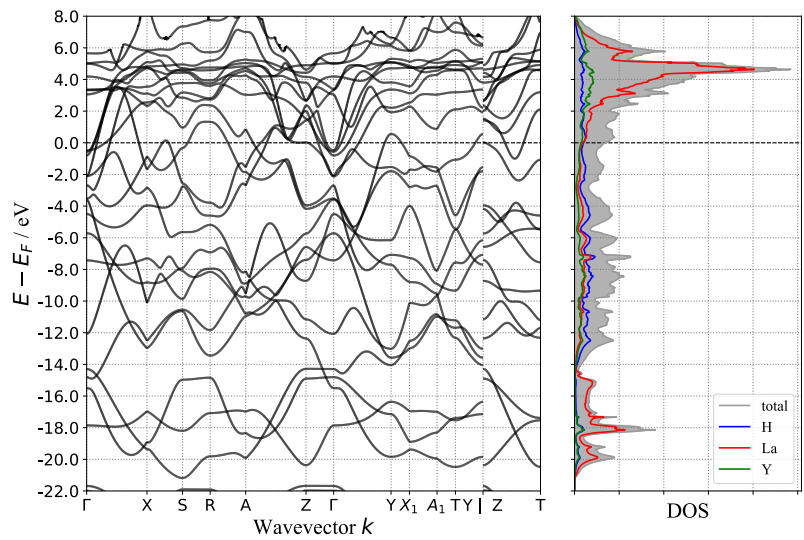

(a)

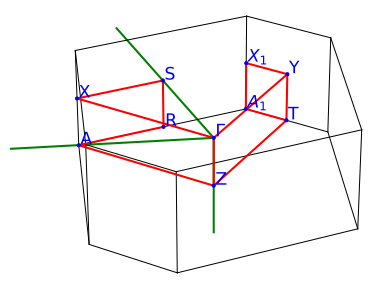

(b)

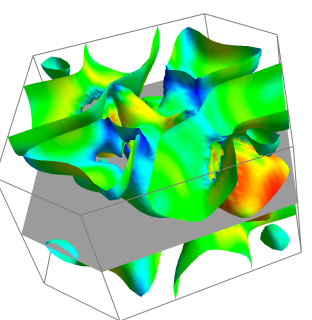

(c)

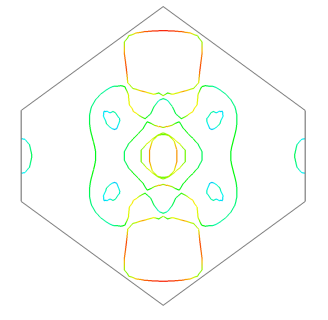

(d)

FIG. 12. Band structure, DOS, and Fermi surface of Cmmm-LaYH $\mathrm{H}_{12}$
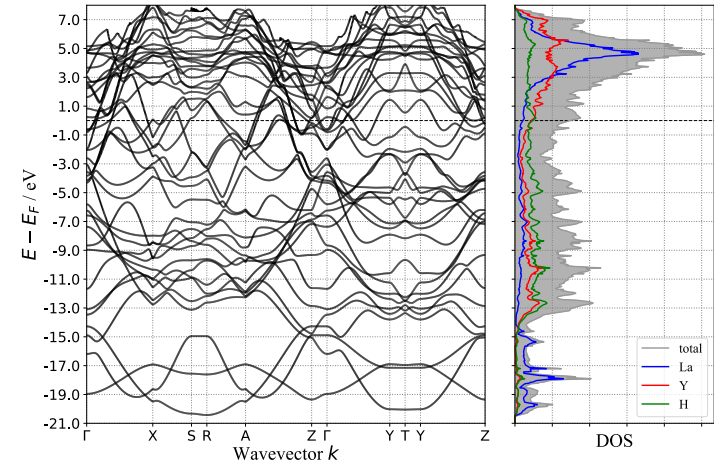

(a)

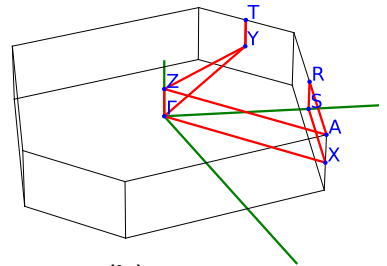

(b)

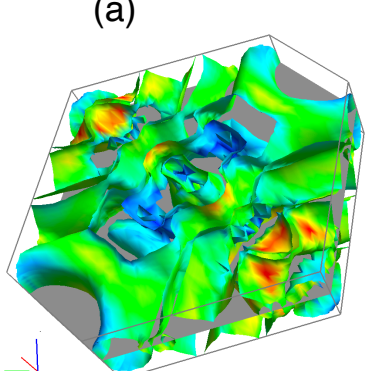

(c)

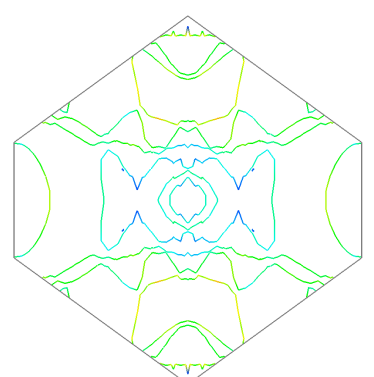

(d)

FIG. 13. Band structure, DOS, and Fermi surface of $\mathrm{Cmmm}-\mathrm{LaY}_{3} \mathrm{H}_{24}$ 


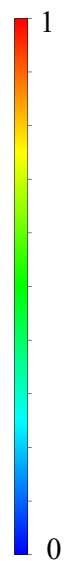

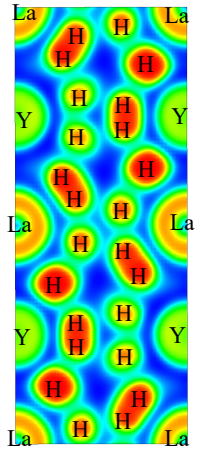

(a)

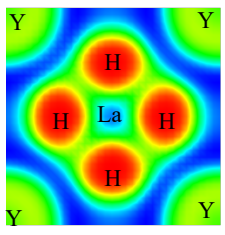

(b)

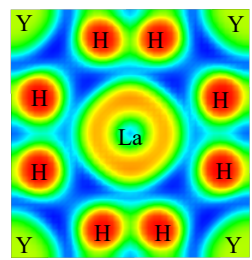

(c)

FIG. 14. The calculated ELF of (a) $R \overline{3} c-\mathrm{LaYH}_{12}$, (b) $P m \overline{3} m-\mathrm{LaYH}_{12}$, and (c) $C m m m-\mathrm{LaYH}_{12}$ in the (100) plane.
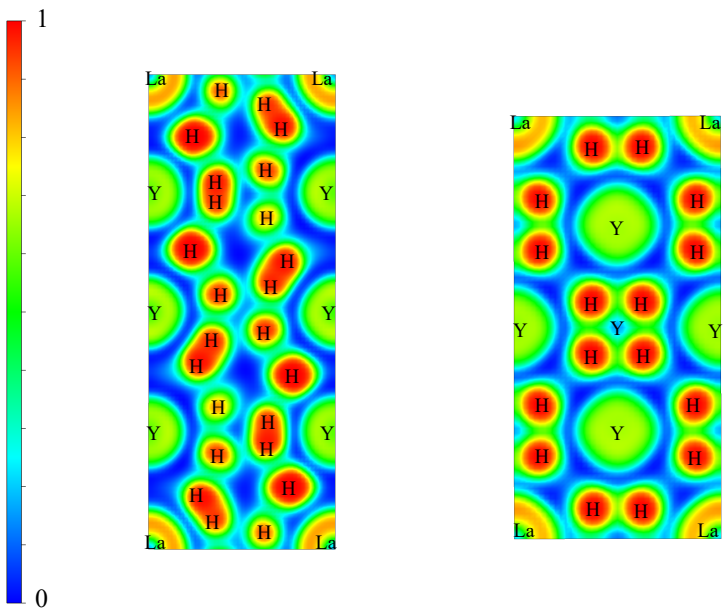

FIG. 15. The calculated ELF of (a) $R \overline{3}-\mathrm{LaY}_{3} \mathrm{H}_{24}$ and (b) $C m m m-\mathrm{LaY}_{3} \mathrm{H}_{24}$ in the (100) plane. 

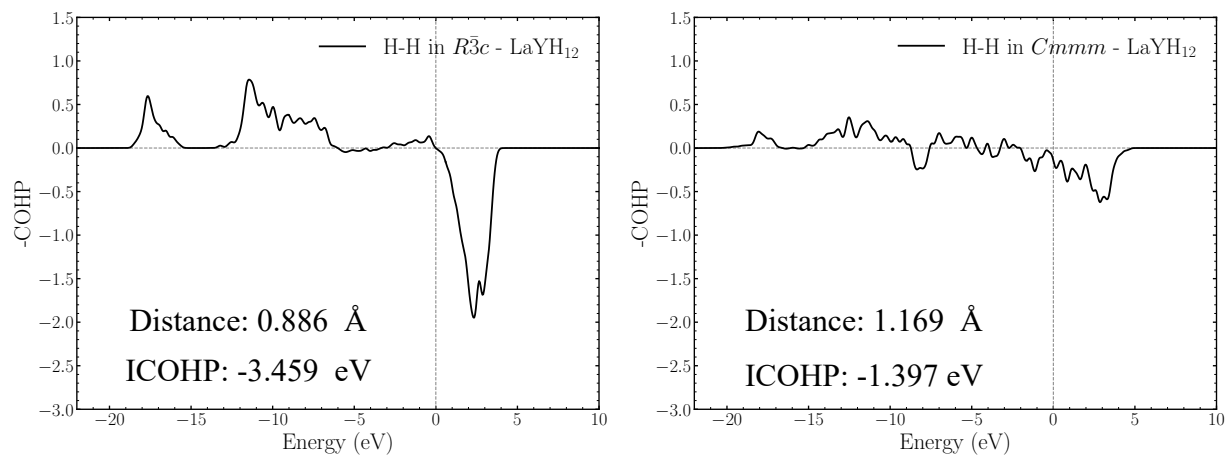

(a)

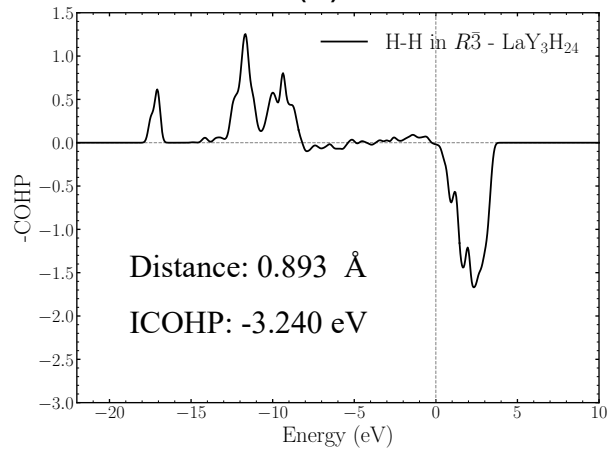

(b)

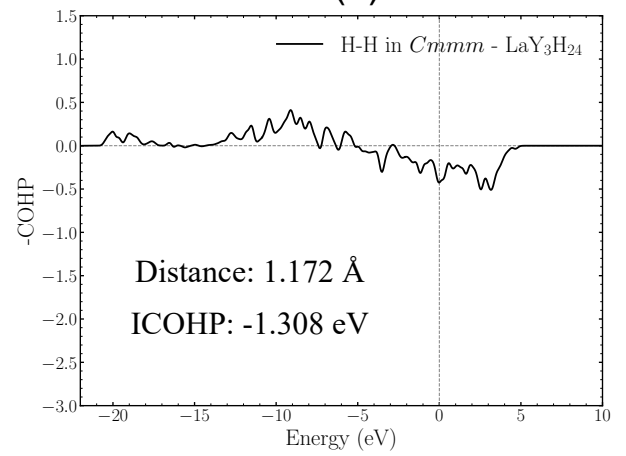

(c)

(d)

FIG. 16. COHP for pairs of $\mathrm{H}-\mathrm{H}$ in (a) $R \overline{3} c-\mathrm{LaYH}_{12}$ at $100 \mathrm{GPa}$, (b) $C m m m-\mathrm{LaYH}_{12}$ at $200 \mathrm{GPa}$, (c) $R \overline{3}-\mathrm{LaY}{ }_{3} \mathrm{H}_{24}$ at $100 \mathrm{GPa}$, and (d) $\mathrm{Cmmm}-\mathrm{LaY}_{3} \mathrm{H}_{24}$ at $180 \mathrm{GPa}$. 